RESEARCH REPORT

\title{
The role of individual and contextual socioeconomic circumstances on mortality: analysis of time variations in a city of north west ltaly
}

\author{
C Marinacci, T Spadea, A Biggeri, M Demaria, A Caiazzo, G Costa
}

J Epidemiol Community Health 2004;58:199-207. doi: 10.1136/jech.2003.014928

See end of article for authors' affiliations

Correspondence to: Dr C Marinacci, Via Sabaudia 16410095 Grugliasco, Turin, Italy; chiara.marinacci@ epi.piemonte.it

Accepted for publication 6 November 2003

\begin{abstract}
Study objective: To evaluate the independent and mutual effects of neighbourhood deprivation and of individual socioeconomic conditions on mortality and to assess the trends over the past 30 years and the residual neighbourhood heterogeneity.

Design: General and cause specific mortality was analysed as a function of time period, highest educational level achieved, housing conditions, and neighbourhood deprivation, using multilevel Poisson models stratified by gender and age class.

Setting: The study was conducted in Turin, a city in north west Italy with nearly one million inhabitants and consisting of 23 neighbourhoods.

Participants: The study population included three cohorts of persons aged 15 years or older, recorded in the censuses of 1971, 1981, and 1991 and followed up for 10 years after each census.

Main results: Individual and contextual socioeconomic conditions showed an independent and significant impact on mortality, both among men and women, with significantly higher risks for coronary heart and respiratory diseases among people, aged less than 65 years, residing in deprived neighbourhoods $19 \%$ and $15 \%$ excess for coronary heart diseases, $20 \%$ and $24 \%$ for respiratory diseases, respectively for men and women living in deprived neighbourhoods compared with rich). The decreasing time trend in general mortality was less pronounced among men with lower education and poorer housing conditions, compared with their more advantaged counterparts; the same was found in less educated women aged less than 65 years.

Conclusions: These results and further developments in the evaluation of impact and mechanisms of other contextual effects can provide information for both health and non-health oriented urban policies.
\end{abstract}

M onitoring the geographical distribution of health status within a city is essential for detecting avoidable heterogeneity and its sources. Variations in health among neighbourhoods can be closely related to differences in their composition in terms of individual characteristics, which consist of specific types of behaviour and risk factors, including, or influenced by, individual socioeconomic conditions. However, variations in health cannot be totally explained by compositional characteristics and could in part be related to the characteristics of the area itself (that is, contextual characteristics). ${ }^{1}$

Although a number of recent studies have investigated heterogeneity in health among neighbourhoods and the impact of neighbourhood level socioeconomic conditions on mortality, morbidity, risk factors, and behaviour, ${ }^{2}$ the existence of an actual neighbourhood effect, the different magnitude of its effect on different causes of deaths within broad age groups, and its underlying mechanisms remain to be determined. Moreover, it is not known whether the finding of a neighbourhood effect in a given city could be generalised to cities as a whole, in that the observed contextual conditions and the magnitude of their effects on health mainly depend on the specific context (that is, affecting certain population groups in certain types of areas)..$^{3}$

The research on the role of contextual characteristics has been recently stimulated by the development of advanced statistical methods, such as multilevel modelling, ${ }^{45}$ which can be used to analyse geographically nested data in the attempt to disentangle the independent and mutual effects of compositional and contextual characteristics. The advantages of these methods lie in the fact that intra-cluster correlation among individuals can be taken into account, providing more appropriate statistical significance of the effects being evaluated. Moreover, the implementation of these methods is facilitated by specific software..$^{6-8}$

In Italy, there is evidence of individual and small area socioeconomic gradients in mortality, mainly for populations living in large cities.9-12 Some preliminary analyses of neighbourhood variations in mortality, adjusting for compositional socioeconomic variability, have highlighted the presence of a residual geographical heterogeneity. ${ }^{12}$

The objective of this study was to evaluate the independent and mutual effects of neighbourhood deprivation and of individual socioeconomic conditions on mortality, to assess the time trends over the past 30 years and the residual neighbourhood heterogeneity in the city of Turin, Italy.

\section{METHODS}

The study was conducted in Turin, a city of about one million inhabitants located in north west Italy. The study population was selected from that of the Turin longitudinal study, ${ }^{13}$ which includes census data for all persons who resided in Turin according to at least one of the last three decennial censuses (1971, 1981, and 1991). For each person, the Turin longitudinal study records the census socio-demographical information and collects information on vital status, cause of death, hospital admissions, the incidence of cancer and diabetes, and drug prescriptions, obtained through record linkage with the local population registry and other local health information systems. The Turin longitudinal study database currently includes records for 2003265 persons. 
This analysis was conducted on three cohorts, which consisted of those persons aged 15 years or older who were recorded in the censuses of $1971(n=799564), 1981$ $(\mathrm{n}=889432)$, and $1991(\mathrm{n}=821 \mathrm{736})$; the first two cohorts were followed up for the subsequent 10 years, while we were able to follow up the third cohort until December 1999.

To represent individual socioeconomic conditions, we used the census data on: (1) highest educational level achieved (high school or college, middle school, primary school or less), as a proxy for social position, and (2) a composite index of housing conditions (that is, large dwelling; small dwelling-owned; small dwelling-rented; and dwelling with no indoor bathroom or heating), used as a proxy of individual economic resources.

For each person, the neighbourhood of residence at the time of the census was taken into account (a total of 23 administrative neighbourhoods and a median number of 41139 inhabitants). A neighbourhood deprivation index ${ }^{14} 15$ was computed based on individual conditions of deprivation in terms of educational resources, economic status, and family support, specifically: low educational level (that is, primary school or less), manual job, rented dwellings, dwellings without an indoor bathroom, household crowding, and single parent households with children. The index was computed by cumulating the standardised scores of each of these conditions at the neighbourhood level; the neighbourhood scores were then classified into five categories, according to quintiles, which were then further collapsed into three groups for the multivariate analysis (advantaged = quintiles I and II, medium = quintile III, and deprived $=$ quintiles IV and V).

Within each stratum defined by specific gender and broad age group, general and cause specific mortality during each of the three cohort periods (that is, 1971-80, 1981-90, and 1991-99) was analysed through multilevel models, with Poisson response and logarithmic link function: firstly, it was modelled as a function of time period and neighbourhood deprivation, controlling for individual demographical characteristics, that is, age class (15-39 and 40-64 for people in the $15-64$ age group, $65-7475-84$, and $\geqslant 85$ years for people aged 65 years or more ) and place of birth (that is, northern Italy, central Italy, southern Italy and the islands, and abroad); secondly, individual education and housing conditions were introduced as covariates in the models (see appendix for a description of the models fitted, for example, for people aged 15-64 years). The estimation procedure was based on second order penalised quasi-likelihood method, with fitting obtained through restricted iterative generalised least squares"; an extra Poisson individual variation was included in the models to allow for the over-dispersion of mortality among individuals within a given neighbourhood. Variations among the three time periods in the effects of individual and contextual socioeconomic conditions on general mortality, were tested using an approximate Wald test ${ }^{4}$ on the related interaction terms: when the test was significant, specific models were fitted, and the coefficient for the time period effect was estimated within each stratum of the given socioeconomic variable. Moreover, significance of random effects was evaluated using the approximate Wald test; this test was also used to assess significance of variations in the effect of individual socioeconomic conditions by level of neighbourhood deprivation, through testing on the related interaction terms.

We finally looked at patterns of neighbourhood deprivation effect, on mortality from specific causes more related to socioeconomic determinants: multilevel models were fitted on mortality by diabetes, stomach cancer, lung cancer, psychological discomfort (AIDS, overdose, psychiatric diseases, suicides, homicides), coronary heart diseases, cerebro- vascular diseases, and respiratory diseases. First mortality was modelled as a function of time period, neighbourhood deprivation, age and place of birth, then individual education and housing conditions were introduced in the models (see appendix).

Statistical analyses were performed using SAS System ${ }^{8}$ and MLwiN ${ }^{6}$ software packages.

\section{RESULTS}

Figure 1 shows the distribution of the study population by educational level, separately within the age groups 15-64 years and $\geqslant 65$ years, and for the three study cohorts. For both age groups, the percentage of persons with the highest educational level increased over time, although the increase was obviously less pronounced among the older persons. There was also a similar improvement in the housing conditions (data not shown), with a particularly noticeable increase in the percentage of persons living in a large dwelling and a decrease in the percentage of persons living in a dwelling with no indoor bathroom or heating.

Figure 2 shows the cumulative mortality rates (standardised by age and gender) for all causes of death in Turin's 23
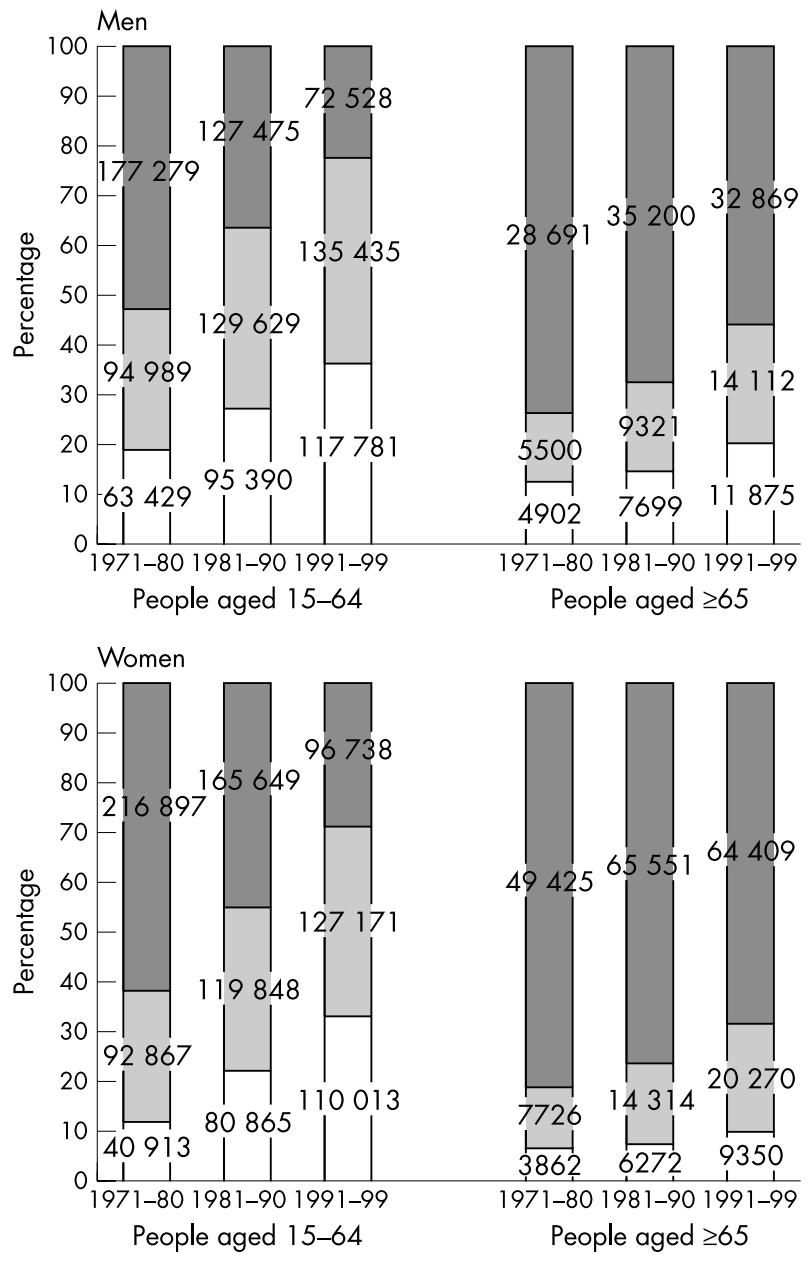

High school or college Middle school Primary school or less

Figure 1 Number of people (\%) by educational level in the three study cohorts (respectively consisting of resident people aged more than 15 years in 1971 and followed up to 1980, resident people aged more than 15 years in 1981 and followed up to 1990, resident people aged more than 15 years in 1991 and followed up to 1999). 


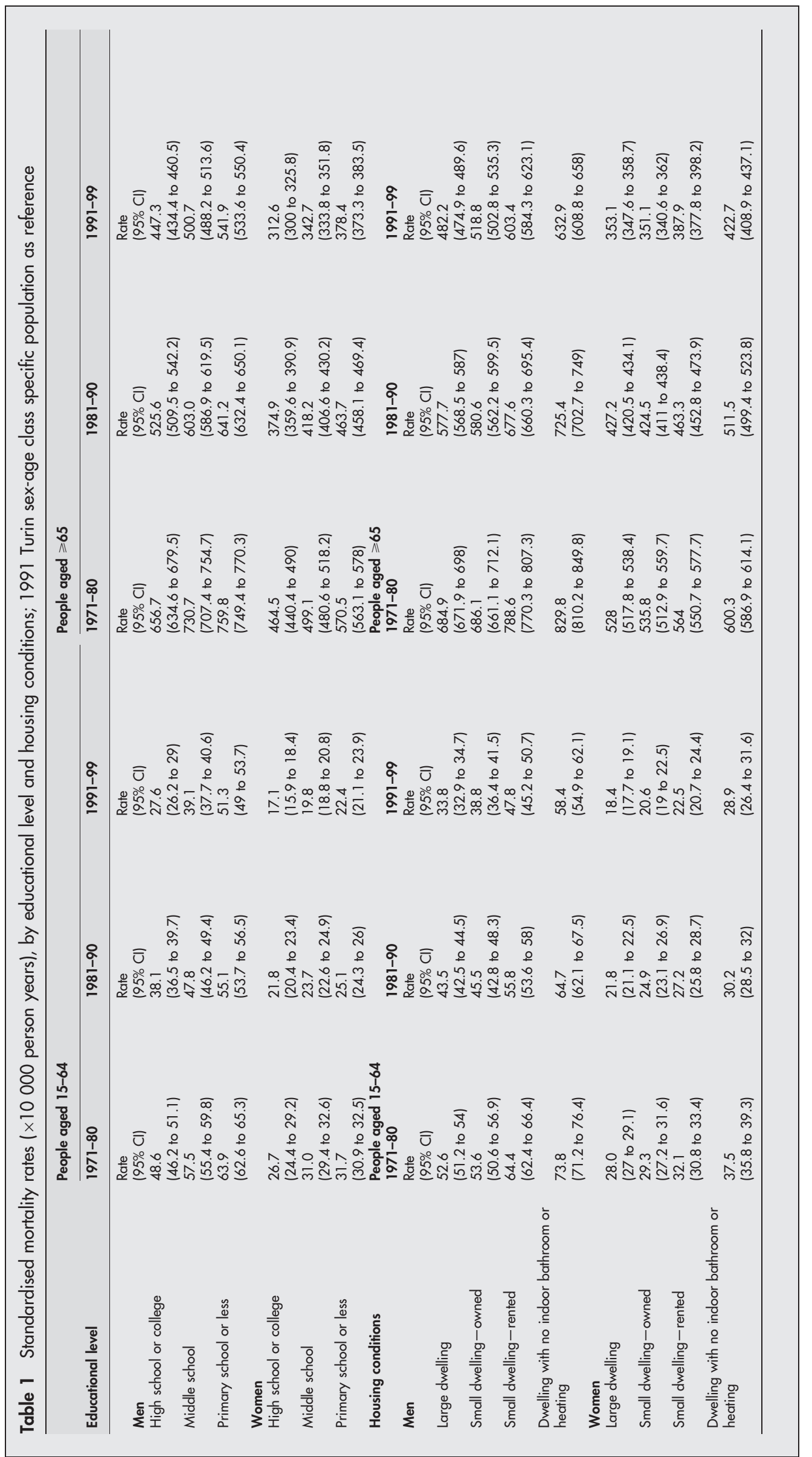




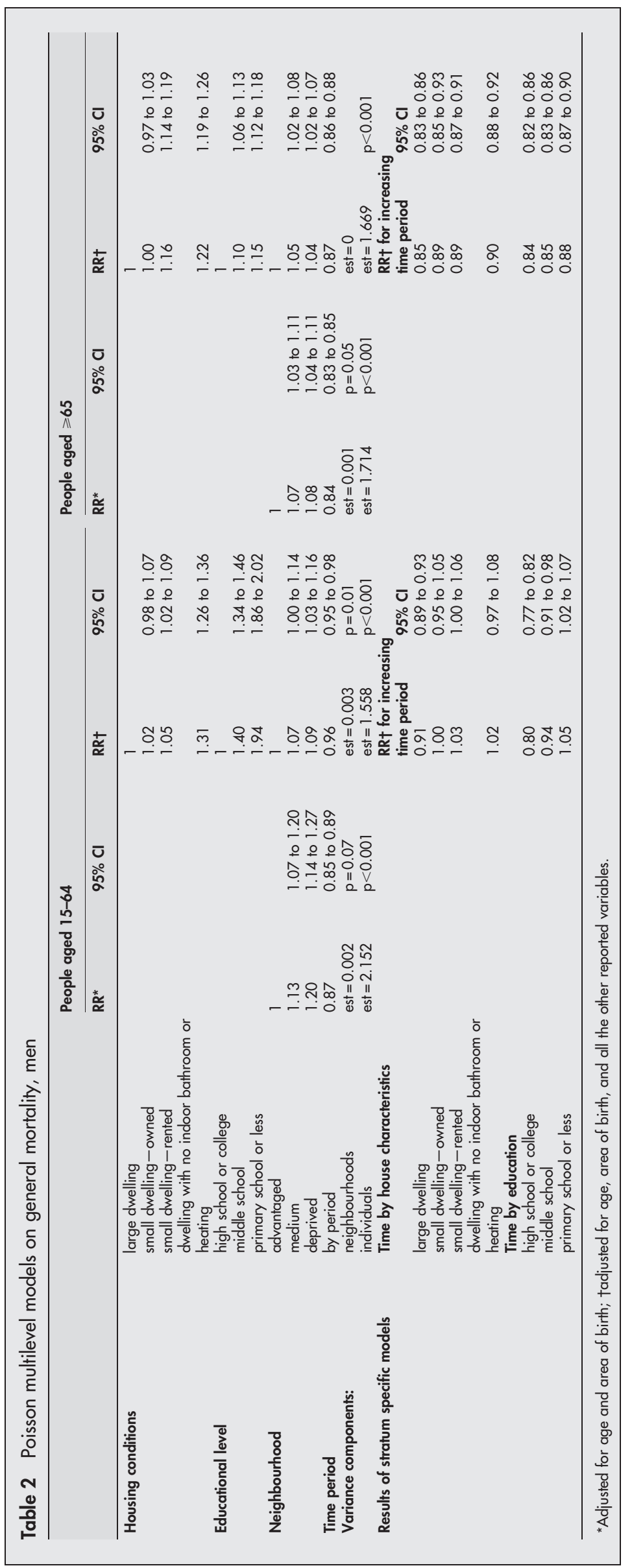




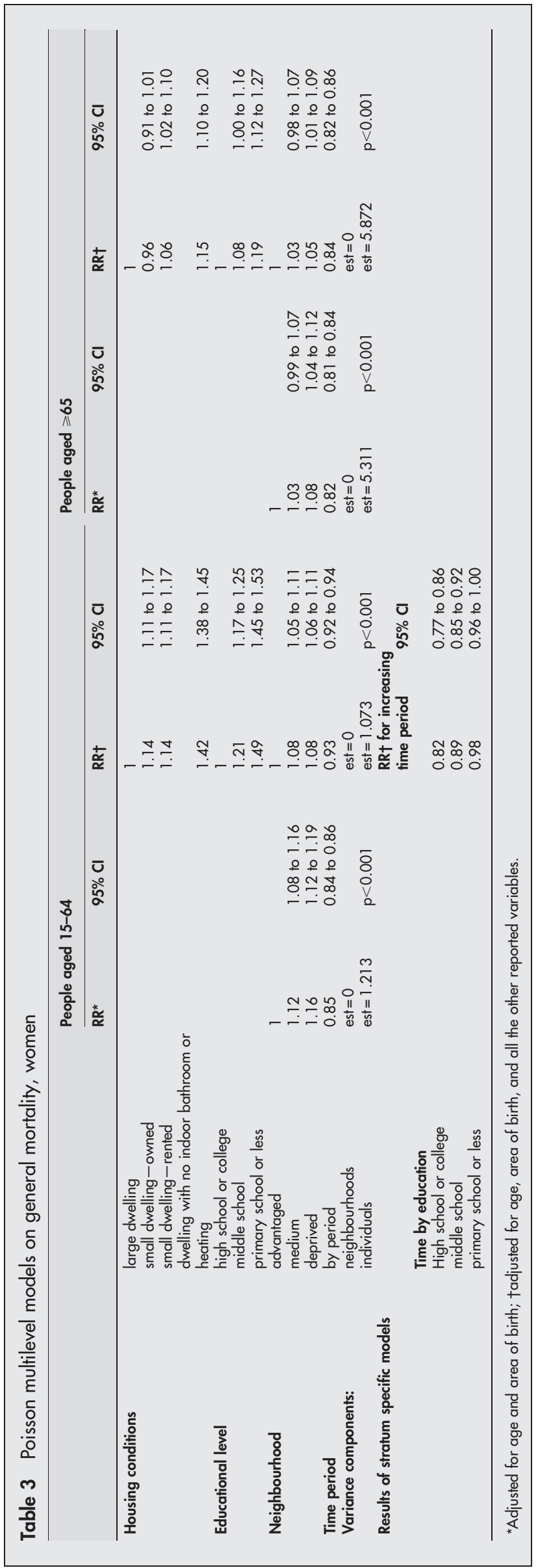

neighbourhoods, for the three periods considered. Mortality tended to be higher in the neighbourhoods of central and northern Turin, with respect to the other areas.

This distribution is very similar to that of the deprivation index, with the poorer neighbourhoods located in northern and central Turin and in the city's southern most point (fig 3). This correlation between mortality and deprivation could in part be the result of differences in the composition of the neighbourhoods in terms of individual socioeconomic conditions, which also seem to affect the overall mortality, as shown in table 1. Mortality increased with decreasing educational level, among both sexes, although the increase was less pronounced for women in the 15-64 year age group in the first two cohorts. A similar profile was observed for housing conditions, although for persons aged $\geqslant 65$ years there was a slight difference in mortality between those who lived in a large dwelling and those living in a small owned dwelling.

Tables 2 and 3 show the relative risk of general mortality, as determined by Poisson multilevel models (level 1 units: individuals; level 2 units: neighbourhoods). Mortality was significantly higher among persons living in more deprived neighbourhoods, for both men and women in both age groups; a slightly but significantly higher risk of death in deprived neighbourhoods was observed even after adjustment for individual socioeconomic characteristics (that is, housing conditions and educational level), which seem more important in the younger group. In the top part of the table we reported results of the models without interactions, while in the lower part we reported estimates of time effects as obtained through models fitted within each single category of socioeconomic indicator with which time interacts. We observed a decreasing trend in mortality over the three periods considered; the extent of this decrease was similar by level of neighbourhood deprivation, yet among men and among younger women it differed by individual socioeconomic conditions. Specifically, among men with poorer housing conditions, the decrease in mortality over time was less pronounced, or even absent among the younger group, compared with that for persons with better housing; similar results were obtained for educational level, with a reversed mortality trend among the younger, and a less pronounced decrease over time in the older, among the less educated men $(\mathrm{p}<0.01$ for both time by education and time by housing conditions interaction terms). Among women in the 15-64 age group, no decrease in mortality was observed among the less educated.

Non-significant interaction terms were found between neighbourhood deprivation and time period, housing conditions, and educational level; thus the effect of neighbourhood deprivation on mortality was steady over time and similar across individual socioeconomic conditions.

Figures 4 and 5 show the relative risks of mortality, as determined by Poisson multilevel models, from specific causes, for which a significant effect of neighbourhood deprivation was found in at least one age-gender specific group. Men aged between 15 and 64 years and living in deprived neighbourhoods showed a significantly higher risk of death for coronary heart disease or respiratory diseases, compared with people living in more advantaged neighbourhoods, even when controlling for individual socioeconomic circumstances. For women in this age class, where a higher proportion of the effect of area deprivation is explained by individual socioeconomic conditions, a significant effect of neighbourhood conditions persisted in those causes and, though very slight, on cerebrovascular diseases. For older people, the negligible effect of neighbourhood on causes of death disappeared, in a large extent, after controlling for compositional effect. 

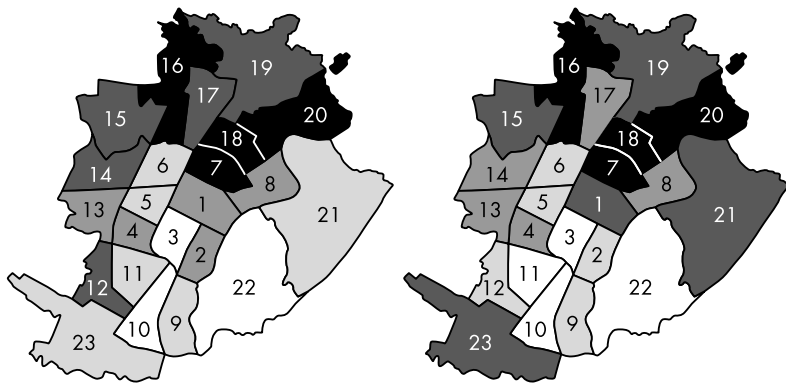

\begin{tabular}{|c|}
\hline Period 1971-1980 \\
$\square \quad 163.5-172.3$ \\
\hline$\square \quad 172.4-176.6$ \\
\hline$\square 176.7-182.5$ \\
\hline$\square \quad 182.6-189.2$ \\
$189.3-200.2$ \\
\hline
\end{tabular}

\section{DISCUSSION}

The distribution of mortality among the neighbourhoods of Turin closely reflects that of deprivation, with higher rates in more deprived areas. This correlation can be explained, in part, by the fact that the more deprived areas have a higher concentration of less educated people (as in the industrialised neighbourhoods of northern Turin and the extreme southern part of the city) or they are characterised by poor housing conditions (as in the case of central Turin). By contrast, the more affluent neighbourhoods of south east and south west Turin have the lowest mortality.

The results also suggest that both compositional and contextual socioeconomic characteristics play a part in the correlation between mortality and area deprivation. When applying the multilevel models, which took into account socioeconomic conditions as both compositional characteristics (that is, educational level and housing conditions) and contextual characteristics (that is, neighbourhood deprivation), the effects of both types of conditions were significant for mortality from all causes and, for people aged less than 65 years, from specific causes. The reduction in the effect of neighbourhood deprivation seems to be relevant, after including compositional socioeconomic characteristics in the model, especially in the younger group, but not so pronounced to exclude the contextual effect of socioeconomic conditions. However, in the $65+$ age group this is observed only for general mortality.

We also observed a non-uniform decreasing trend in mortality among men and younger women; specifically, with

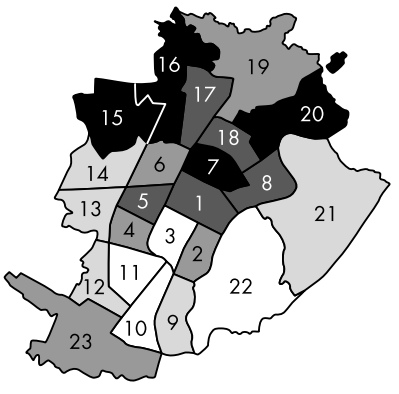

Figure 2 Deaths from all causes by neighbourhood of Turin. Quintiles of rates ( $\times 10000$ person years) standardised by age and gender, with 1991-1999 person years as reference. (Study cohorts respectively consisting of resident people aged more than 15 years in 1971 and followed up to 1980, resident people aged more than 15 years in 1981 and followed up to 1990, resident people aged more than 15 years in 1991 and followed up to 1999).

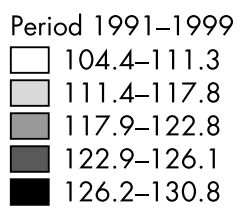

a less evident decrease in mortality among those who were more deprived in terms of individual socioeconomic conditions compared with the more advantaged counterparts. Inequalities in mortality according to individual socioeconomic conditions are widening, as observed in most studies conducted in past years in other countries. ${ }^{16}$ In general, this may be attributable to more rapid improvements in life expectancy among the well off component of society (less strained, more capable of adopting a healthy lifestyle, and of using health services). By contrast, among older women individual inequalities in mortality have not significantly changed over time, because of the slower improvement in health of the more educated, as from the spread of the "smoking epidemic"17 and from the lower fertility, with related cancer mortality, among this group. Moreover, changes in time period effect, before and after inclusion of compositional socioeconomic conditions, seem to highlight that much of the decreasing trend in mortality among younger men and women can be explained by changes in socioeconomic circumstances of the population over time. We also observed that the slight and independent role of contextual deprivation has not been changing over time.

Previous attempts to disentangle the effects of compositional and contextual characteristics at the neighbourhood level seem to indicate that contextual characteristics do have an effect on health, although whether or not this effect is independent of compositional characteristics remains to be determined. ${ }^{18-22}$ However, in our study,

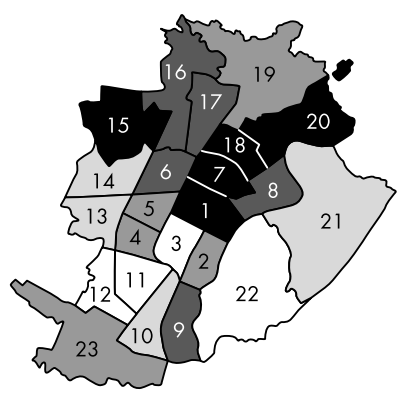

1971

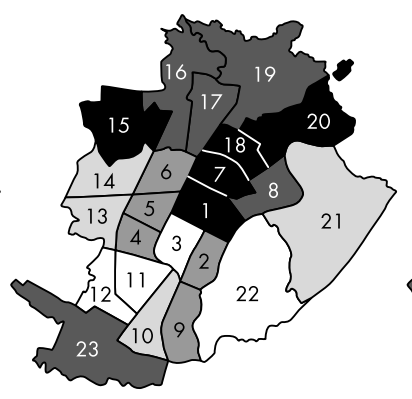

1981

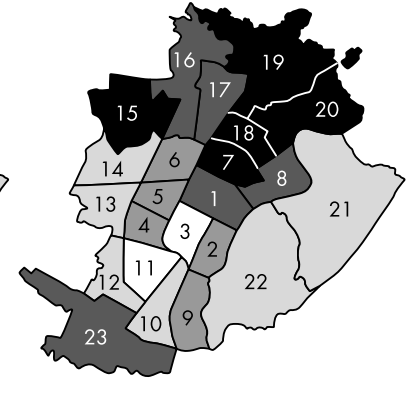

1991
Figure 3 Neighbourhoods by deprivation level. All residents in Turin in 1971, 1981, and 1991 censuses. (Study cohorts respectively consisting of resident people aged more than 15 years in 1971 and followed up to 1980 , resident people aged more than 15 years in 1981 and followed up to 1990 , resident people aged more than 15 years in 1991 and followed up to 1999).

\footnotetext{
Very advantaged Advantaged Medium Deprived Very deprived
} 


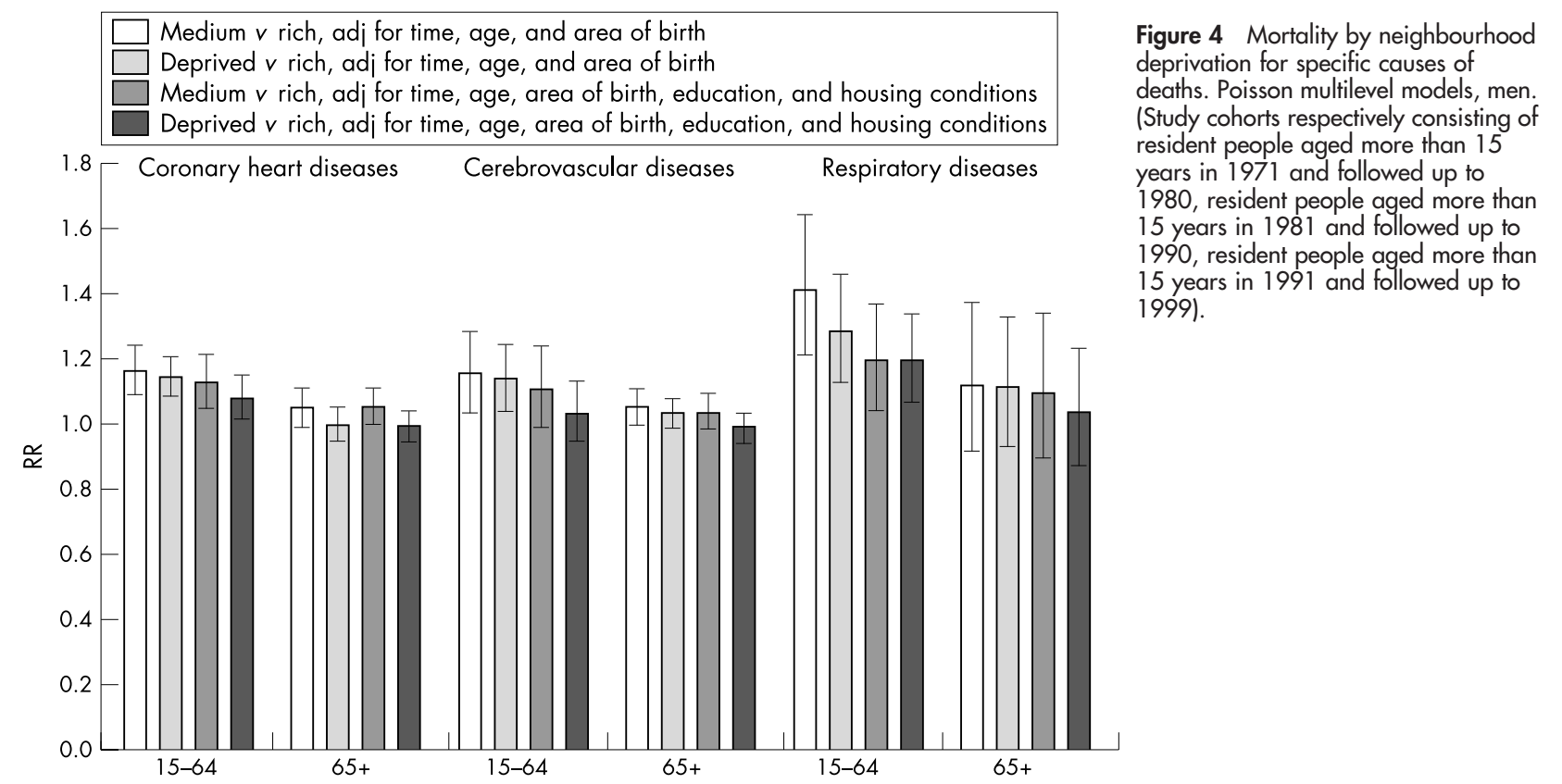

we failed to find any significant interaction between neighbourhood deprivation and individual socioeconomic variables.

In the attempt to identify the mechanisms underlying the effect of contextual deprivation, findings published so far have highlighted an impact on many stages of the natural history of disease and the pathway towards death. For example, area deprivation has been found to be associated with an increased prevalence of at risk behaviour, such as smoking ${ }^{23-25}$ and unhealthy diet. ${ }^{26}$ It has also been shown to affect morbidity, as determined by self reported health, long term illness, and chronic conditions, ${ }^{27-29}$ and low birth weight, ${ }^{30} 31$ even after controlling for individual confounders. Studies on cause specific mortality and the occurrence of specific diseases have provided clues as to the specific action of neighbourhood deprivation on health. For example, area deprivation has been consistently shown to have an effect not only on the occurrence of coronary heart disease but also on the risk factors related to this type of disease, with the patterns at times varying by gender and ethnicity. ${ }^{26}{ }^{32-37}$ Our results on cause specific mortality are consistent with this description in the 15-64 age group, with the additional relevance of respiratory diseases shown in the Turin context. Anyway the causal mechanisms for a neighbourhood effect on specific health problems still need to be thoroughly evaluated; in an attempt to better understand these mechanisms, one study identified five groups of contextual factors that can worsen the health of persons living in disadvantaged neighbourhoods: physical features of the environment, quality of housing and of the working environment, the services provided for life of the residents (for example, health care and transportation), sociocultural

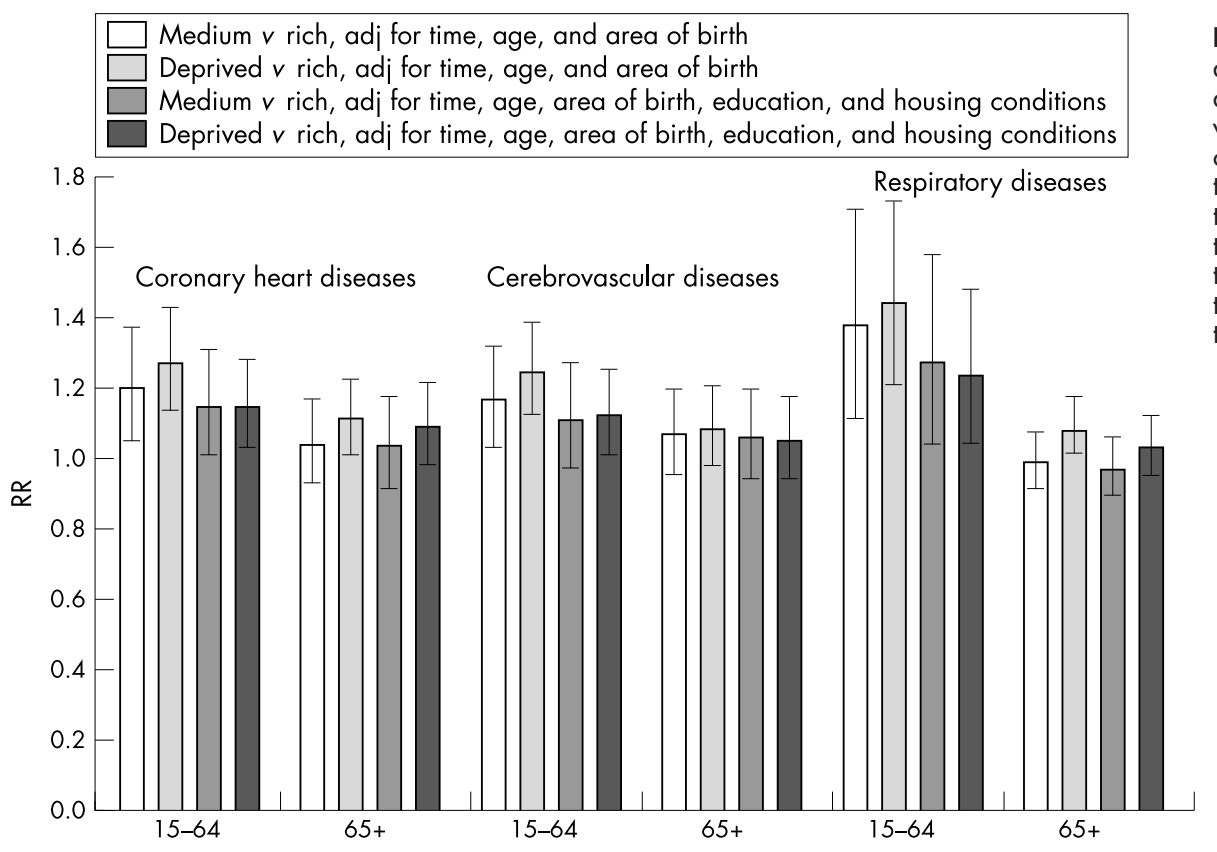

Figure 5 Mortality by neighbourhood deprivation for specific causes of deaths. Poisson multilevel models, women. (Study cohorts respectively consisting of resident people aged more than 15 years in 1971 and followed up to 1980 , resident people aged more than 15 years in 1981 and followed up to 1990 , resident people aged more than 15 years in 1991 and followed up to 1999). 


\section{Key points}

- Variations in health among neighbourhoods can be partly related to differences in their composition in terms of individual socioeconomic characteristics, but it has been suggested that they could also be produced by the characteristics of the area itself.

- We observed that both individual and contextual socioeconomic conditions had an independent and significant impact on mortality in the city of Turin, in both sexes, with significantly higher risks for coronary heart and respiratory diseases among people, aged less than 65 years, residing in deprived neighbourhoods.

- The decrease in mortality over the past 30 years was less pronounced among men and women with a lower educational level and among men with poorer housing conditions, compared with their more advantaged counterparts; by contrast, the effect of contextual deprivation has not been changing over time.

features, and the reputation of an area; a classification based on universal human needs could support the operational definition of indicators aimed at measuring the health impact of these dimensions.

We considered place of birth as a compositional characteristic: this represents a strong confounder in the association between health and socioeconomic characteristics in Turin, as this city has for nearly half a century experienced a great influx of persons immigrating from other parts of Italy, especially from southern Italy, whose native population has been shown to be healthier than the population of Turin ${ }^{38}$ and more deprived. However, our finding of a slight residual geographical variability in general mortality among younger men, even after controlling for individual and contextual variables, may have been attributable to misclassification or to the failure to take into consideration other important compositional determinants, as for example occupational exposures, or contextual characteristics, like public housing urban interventions affecting selective residential mobility.

Inferences on neighbourhood level effects usually present methodological challenges. One of them is the choice of boundaries for level 2 units: the selection of administrative neighbourhoods is debatable, ${ }^{29}$ in that it assumes that there is plausible and measurable variation in contextual characteristics at this area level. Our choice of administrative boundaries was based not only on the availability of data but also on the knowledge that, in Turin, a given neighbourhood is, to a large extent, socially, culturally, and historically homogeneous. The neighbourhood distribution of the Turin population has been, in fact, strongly produced by the expansion and by the allocation of the industrial production during the 20th century: this has determined the stratification of successive migratory waves and the socioeconomic characterisation of areas (even if irregularly over time). ${ }^{40}$ Inferences regarding the deprivation effect at this level can involve factors that are relevant to health at this geographical level (for example, the availability of services, quality of life, and safety); they can also be important in supporting certain urban area policies.

It could be argued that the effect of neighbourhood deprivation that we observed was diminished by having controlled for individual housing conditions, which may have captured part of the contextual effect, in that housing
Policy implications

- Inferences regarding the neighbourhood socioeconomic deprivation effect can be important in supporting urban policies.

- Future developments in the evaluation of the operating mechanisms underlying the effect of aggregate deprivation and in the assessment of the impact of other contextual characteristics, will provide important elements for both health and non-health oriented urban policies for reducing or eliminating the exposure to health hazards.

- The massive recruitment of labourers carried out by Turin industries from 1950s to 1970 s was not accompanied by any programmed territorial initiatives that took into account adequate health and social services. Only recently the most critical neighbourhoods are subject to comprehensive community involvement policies, based on improvement of labour, social, and healthcare services.

- This study offers an example of the available tools for the evaluation of the impact of these policies and to stimulate cooperation among actors within different public and private institutions.

conditions are the same within a building or blocks of buildings in the same area. None the less, the area on which we based the contextual deprivation index is large enough that we can assume that the two sets of information refer to independent dimensions.

Given the nature of our study design, we were not able to take into account the impact of residential changes over lifetime and the cumulative effects of socioeconomic environment over the entire study period. Notwithstanding these and other limitations, we believe that this study and similar studies can provide important information for developing more than just local health policies; in fact, developments in the evaluation of the impact of other contextual characteristics and in the investigation of the operating mechanisms underlying the effect of aggregate deprivation on health are important elements for urban policies having an impact on health. Particularly in the extreme south and north areas of the town, the massive recruitment of labourers carried out by industries from 1950s to 1970s was not accompanied by any programmed initiatives for adequate health and social services within territory, in order to answer to the health and well being needs of the new labourers; this lasted until the 1990s, with the exception of sporadic interventions. ${ }^{41}$ Only recently the most critical neighbourhoods are subject to comprehensive community involvement policies, based on enhancement of labour, social, and healthcare services.

\section{ACKNOWLEDGEMENTS}

We wish to acknowledge Mark Kanieff for editorial assistance. In developing the methodology of the study, we greatly benefited from the contributions provided by the participants of the 2nd joint meeting of WGI and WGII of the European Science Foundation Scientific Program "Social Variations in Health Expectancy in Europe"

\section{Authors' affiliations}

C Marinacci, T Spadea, A Caiazzo, Epidemiology Unit, Piedmont Region, Italy

A Biggeri, Department of Statistics G Parenti, University of Florence, Italy 
M Demaria, Unit of Environmental Epidemiology, Piedmont Region, Italy G Costa, Department of Public Health and Microbiology, University of Turin, Italy

Funding: this study was supported by the 1998 Research Programme of the Ministero della Salute - "Programmi speciali" (ex art 12c 2 left B) D Lgs 502/92 - under contract named "Un sistema informativo, un librobianco, un modello di monitoraggio per implementare gli obiettivi di equità nella salute e nell'assiotonza in Italia".

Conflicts of interest: none declared.

\section{APPENDIX}

As an example, for people in the 15-64 age group, the models fitted were:

\section{STEP 1}

$\log \pi_{\mathrm{ij}}=$ offset $+\beta_{1 \mathrm{j}}+\beta_{2}$ time $_{\mathrm{ij}}+\beta_{3}$ age $40-64_{\mathrm{ij}}+\beta_{4}$ borne in centre ${ }_{\mathrm{ij}}+\beta_{5}$ borne in south/isle ${ }_{\mathrm{ij}}+\beta_{6}$ borne abroad ${ }_{\mathrm{ij}}+\beta_{7}$ medium neighbourhood $_{j}+\beta_{8}$ deprived neighbourhood ${ }_{j}$ where

$$
\begin{aligned}
& \beta_{\mathrm{lj}}=\beta_{\mathrm{l}}+\mathrm{u}_{\mathrm{lj}} \\
& \text { deaths }_{\mathrm{ij}} \sim \text { Poisson }\left(\pi_{\mathrm{ij}}\right) \\
& \text { deaths }_{\mathrm{ij}}=\pi_{\mathrm{ij}}+\varepsilon_{\mathrm{ij}} \\
& \mathrm{u}_{\mathrm{lj}} \sim \mathrm{N}_{\left(0, \sigma^{2}{ }_{\mathrm{ul}}\right)} \\
& \varepsilon_{\mathrm{ij}} \sim\left(0, \sigma^{2}{ }_{\mathrm{e}}\right)
\end{aligned}
$$

\section{STEP 2}

$\log \pi_{\mathrm{ij}}=$ offset $+\beta_{1 \mathrm{j}}+\beta_{2}$ time $_{\mathrm{ij}}+\beta_{3}$ age $40-64_{\mathrm{ij}}+\beta_{4}$ borne in centre ${ }_{\text {ij }}+\beta_{5}$ borne in south/isle ${ }_{i j}+\beta_{6}$ borne abroad ${ }_{i j}+\beta_{7}$ medium neighbourhood $_{\mathrm{j}}+\beta_{8}$ deprived neighbourhood ${ }_{\mathrm{j}}+\beta_{9}$ middle school $_{\mathrm{ij}}+\beta_{10}$ primary school or less $\mathrm{ij}_{\mathrm{ij}}+\beta_{11}$ small dwelling owned ${ }_{i j}+\beta_{12}$ small dwelling rented $_{i j}+\beta_{13}$ no toilet $t_{i j}$ where

$\beta_{1 j}=\beta_{1}+u_{1 j}$

deaths ${ }_{\mathrm{ij}} \sim$ Poisson $\left(\pi_{\mathrm{ij}}\right)$

deaths $_{\mathrm{ij}}=\pi_{\mathrm{ij}}+\varepsilon_{\mathrm{ij}}$

$\mathrm{u}_{1 \mathrm{j}} \sim \mathrm{N}\left(0, \sigma_{\mathrm{ul}}^{2}\right)$

$\varepsilon_{\mathrm{ij}} \sim\left(0, \sigma_{\mathrm{e}}^{2}\right)$

\section{REFERENCES}

1 Macintyre S, Maciver S, Sooman A. Area, class and health; should we be focusing on places or people? Journal of Social Policy 1993;22:213-34.

2 Pickett KE, Pearl M. Multilevel analyses of neighbourhood socioeconomic context and health outcomes: a critical review. J Epidemiol Community Health 2001:55:111-22.

3 Macintyre S, Ellaway A, Cummins S. Place effects on health: how can we conceptualise, operationalise and measure them? Soc Sci Med 2002;55:125-39

4 Goldstein H. Multilevel statistical models. New York: Halsted Press, 1995.

5 Snijders T, Bosker R. Multilevel Analysis. An introduction to basic and advanced multilevel modelling. London: Sage, 2000:1-86.

6 Rasbash J, Browne W, Healy M, et al. MlwiN Version 1.10.0006. London: Multilevel Models Project, Institute of Education, 2000.

7 Hedeker D, Gibbons RD. MIXREG: a computer program for mixed-effects regression analysis with autocorrelated errors. Comput Methods Programs Biomed 1996;49:229-52.

8 SAS Institute. The SAS system for Windows, release 8.01. Cary, NC: SAS, 1999 .

9 Mackenbach JP, Kunst AE, Groenhof F, et al. Socioeconomic inequalities in mortality among women and among men: an international study. Am J Public Health 1999;89:1800-6

10 Michelozzi P, Perucci CA, Forestiere F, et al. Inequality in health: socioeconomic differentials in mortality in Rome, 1990-95. J Epidemiol Community Health 1999;53:687-93.

11 Costa G, Spadea T, Marinacci C, et al. Looking at health inequalities to identify targets. Ann $\lg 2002 ; 14: 79-86$.
12 Biggeri A, Marchi M, Dreassi E, et al. The Tuscany longitudinal study: mortality among selected causes in inner city of Florence and Leghorn. Epidemiol Prev 1999;23:161-74.

13 Office of National Statistics. Longitudinal study newsletter no 24. London: Office of National Statistics and Centre for Longitudinal Study, University of London, 2001:2-3.

14 Morris R, Carstairs V. Which deprivation? A comparison of selected deprivation indexes. J Public Health Med 1991;13:318-26.

15 Folwell K. Single measures of deprivation. J Epidemiol Community Health 1995;49:S51-6.

16 Mackenbach J, Bos V, Andersen O, et al. Widening socioeconomic inequalities in mortality in six Western European countries. Int J Epidemiol 2003;32:830-7.

17 Faggiano F, Versino E, Lemma P. Decennial trends of social differentials in smoking habits in Italy. Cancer Causes Control 2001;12:665-71.

18 Davey Smith G, Hart C, Blane D, et al. Lifetime socioeconomic position and mortality: prospective observational study. BMJ 1997;22:547-52.

19 Veugelers PJ, Yip AM, Kephart G. Proximate and contextual socio-economic determinants of mortality: multilevel approaches in a setting with universal health care coverage. Am J Epidemiol 2001;154:725-32.

20 Haan M, Kaplan GA, Camacho T. Poverty and health: prospective evidence from the Alameda County Study. Am J Epidemiol 1987;125:989-98.

21 Waitzman N, Smith K. Phantom of the area: poverty-area residence and mortality in the United States. Am J Public Health 1998;88:973-6.

22 Sloggett A, Joshi H. Deprivation indicators as predictors of life events 19811992 based on the UK ONS Longitudinal Study. J Epidemiol Community Health 1998;52:228-33.

23 Kleinschmidt I, Hills M, Elliott P. Smoking behaviour can be predicted by neighbourhood deprivation measures. J Epidemiol Community Health 1995;49:S72-7.

24 Reijneveld SA. The impact of individual and area characteristics on urban socioeconomic differences in health and smoking. Int J Epidemiol 1998;27:33-40.

25 Duncan C, Jones K, Moon G. Smoking and deprivation: are there neighbourhood effects? Soc Sci Med 1999;48:497-505.

26 Van Lenthe FJ, Mackenbach JP. Neighbourhood deprivation and overweight: the GLOBE study. Int J Obes Relat Metab Disord 2002;26:234-40.

27 Shouls S, Congdon P, Curtis S. Modelling inequality in reported long-term illness in the UK: combining individual and area characteristics. J Epidemiol Community Health 1996;50:366-76.

28 Robert SA. Community-level socioeconomic status effects on adult health. J Health Soc Behav 1998;39:18-37.

29 Reijneveld SA. Neighbourhood socioeconomic context and self reported health and smoking: a secondary analysis of data on seven cities. J Epidemiol Community Health 2002;56:935-42.

30 Roberts E. Neighbourhood social environments and the distribution of low birthweight in Chicago. Am J Public Health 1997;87:597-603.

31 Pearl M, Braveman P, Abrams B. The relationship of neighbourhood socioeconomic characteristics to birthweight among 5 ethnic groups in California. Am J Public Health 2001;91:1808-14.

32 Davey Smith G, Hart C, Watt G, et al. Individual social class, area-based deprivation, cardiovascular disease risk factors, and mortality: the Renfrew and Paisley Study. J Epidemiol Community Health 1998;52:399-405.

33 Diez Roux AV, Nieto FJ, Muntaner C, et al. Neighbourhood environments and coronary heart disease: a multilevel analysis. Am J of Epidemiology 1997; 146:48-63.

34 Sundquist J, Malmstrom M, Johansson SE. Cardiovascular risk factors and the neighbourhood environment: a multilevel analysis. Int J Epidemiol 1999;28:841-5.

35 Diez Roux AV, Merkin SS, Arnett D, et al. Neighborhood of residence and incidence of coronary heart disease. N Engl J Med 2001;345:99-106.

36 Hart C, Ecob R, Davey Smith G. People, places and coronary heart disease risk factors: a multilevel analysis of the Scottish Heart Health Study archive. Soc Sci Med 1997;45:893-902.

37 Kolegard Stiarne M, Diderichsen F, Reuterwall C, et al. Socioeconomic context in area of living and risk of myocardial infarction: results from Stockholm Heart Epidemiology Program (SHEEP). J Epidemiol Community Health 2002;56:29-35.

38 Costa G, Cardano M, Demaria M. Torino. Storie di salute in una grande città Torino: Ufficio Statistico città di Torino, 1998:222-36.

39 Diez Roux A. Investigating neighbourhood and area effects on health. Am J Public Health 2001;91:1783-9.

40 Castronovo V. Torino. Torino: Laterza, 1987.

41 Castagnoli A. Le istituzioni locali e le classi dirigenti dal dopoguerra alla metà degli anni Ottanta. In: Tranfaglia N, ed. Storia di Torino. Torino: Einaudi, 2001:103-55. 